Dedicated to Professor J.N. Reddy on the Occasion of His $75^{\text {th }}$ Birthday

\title{
ACCURATE THROUGH-THE-THICKNESS STRESS DISTRIBUTIONS IN THIN-WALLED METALLIC STRUCTURES SUBJECTED TO LARGE DISPLACEMENTS AND LARGE ROTATIONS
}

\author{
A. Pagani ${ }^{1}$, R. Azzara ${ }^{1}$, R. Augello ${ }^{1}$, E. Carrera ${ }^{1}$, B. $\mathbf{W u}^{2}$ \\ ${ }^{1}$ Department of Mechanical and Aerospace Engineering, Politecnico di Torino, Torino, Italy \\ ${ }^{2}$ School of Mathematics, Statistics and Applied Mathematics, NUI Galway, University Road, Galway, Ireland \\ *E-mail: alfonso.pagani@polito.it
}

Received: 09 July 2020 / Published online: 27 September 2020

\begin{abstract}
The present paper presents the evaluation of three-dimensional (3D) stress distributions of shell structures in the large displacement and rotation fields. The proposed geometrical nonlinear model is based on a combination of the Carrera Unified Formulation (CUF) and the Finite Element Method (FEM). Besides, a Newton-Raphson linearization scheme is adopted to compute the geometrical nonlinear equations, which are constrained using the arc-length path-following method. Static analyses are performed using refined models and the full Green-Lagrange strain-displacement relations. The Second PiolaKirchhoff (PK2) stress distributions are evaluated, and lower- to higher-order expansions are employed. Popular benchmarks problems are analyzed, including cylindrical isotropic shell structure with various boundary and loading conditions. Various numerical assessments for different equilibrium conditions in the moderate and large displacement fields are proposed. Results show the distribution of axial and shear stresses, varying the refinement of the proposed two-dimensional (2D) shell model. It is shown that for axial components, a lower-order expansion is sufficient, whereas a higher-order one is needed to accurately predict shear stresses.
\end{abstract}

Keywords: Carrera Unified Formulation, three-dimensional stress field, second Piola-Kirchhoff stress, refined 2D shell theory, geometrical nonlinearity.

\section{INTRODUCTION}

Nowadays, shell structures are increasingly employed in many engineering fields, for instance, in aerospace, for aircraft and spacecraft skins and civil, for bridges and roofs, among the others. Basically, shells consist of curved structures where the thickness is smaller than the other two dimensions and can support twisting, extension, compression, bending, transverse shear, and in-plane shear loads. The geometric characteristics of shell structures, including the initial curvatures, have a direct influence on the stiffness properties [1]. The main advantage of these thin-walled structures is the capability of carrying loads with high efficiency. Carrera et al. [2] described the importance of the adoption of a two-dimensional (2D) model, which is able to accurately describe internal stress states and displacement fields when external loading conditions become large. In fact, shell structures are prone to suffer large rotations. A proper 
design of such structures is crucial to perform accurate stress predictions under different loading conditions. The popularity of the $2 \mathrm{D}$ shell models is thanks to their lower computation cost compared to three-dimensional (3D) models.

The aforementioned reasons led scientists to develop efficient 2D shell models. The classical theories are represented by Poisson [3], Love [4], Mindlin [5], Kirchhoff [6], Reissner [7] and Cauchy [8]. Commercial codes adopt these classical theories $[9,10]$ within their shell elements. As discussed by Petrolo and Carrera [11], it is important to process various parameters, for example, the anisotropy, gradients in the strain and stress fields, thickness ratio, and inhomogeneity to evaluate the accuracy of classical models. The applicability of the classical theories is limited to a narrow range of applications, for example, when dealing with the thinwalled structure and without local effects. By contrast, if the assessment of transverse stresses is important, more attention is needed. Recently, different higher-order $2 \mathrm{D}$ formulations were formulated to improve the accuracy of classical theories. A review of the theories is not the scope of this work, but given the importance of the topic, a brief overview follows. Petrolo and Carrera [11] developed a useful review of methods and guidelines for the choice of shell theories. A parabolic distribution of transverse shear deformations across the thickness was considered by Reddy and Liu [12] in their shear model. A simple high-order theory for laminated composite 2D structures was reported by Reddy [13]. The same author [14], illustrated a review of the mechanics of a laminated composite plate and shell with different analyses. In addition, in the framework of Carrera Unified Formulation (CUF) $[15,16]$ different refined shell theories are studied. Cinefra and Carrera [17] provided finite shell elements with different through-the-thickness kinematics for the linear analysis of cylindrical multilayered structures. Other significant works on refined shell theories can be found in $[18,19]$.

In recent decades, different engineering fields use highly flexible structures. As reported by Pagani et al. [20], these structures are prone to support large displacements and rotations, and snapping phenomena can occur. Consequently, Wu et al. [21] described that an accurate failure prediction is of primary importance in the analyses of the nonlinear response of highly flexible structures in the geometrical nonlinear domain. In the open literature, there are several studies related to higher-order shell models in order to accurately perform geometrical nonlinear analyses. Considerable interest in this topic is evident in modern literature. Recently, different popular nonlinear benchmark problems of shells were suggested by Sze et al. [22]. Palazotto [23] provided many of the features associated with large displacements and rotations within a cylindrical shell, including through-the-thickness shear flexibility. Ma and Wang [24] adopted a classical nonlinear von Kármán plate theory to study circular plates under mechanical and thermal loadings in the large displacement field. The von Kármán theory provides a good approximation for the nonlinear analysis of 2D structures, and for this reason, it was widely adopted by scientists. Carrera and Parisch [25] proved that the von Kármán approximation provides reliable results in the study of thin shell structures, but not for thick ones. A nonlinear finite element (FE) formulation for the quasi-static analysis of shells in the large strains and rotations field is implemented by Hughes and Liu [26]. Surana [27] analyzed 3D curved shell elements using a Total Lagrangian (TL) method. Lee and Kanouk-Nukulchai [28] adopted the arc-length method to catch any load-deflection curves for isotropic and composite shells in the large deformation domain. A new shell element able to remove locking phenomena in the geometrical nonlinear analysis was investigated by Ko et al. [29]. Many applications for capturing accurate 3D stresses in shell-like structures were conducted [30], and a number of stress definitions were developed in the last decades. For example, Tortorelli [31] used the Piola-Kirchhoff stress and the Cauchy stress formulation to perform a sensitivity analysis for nonlinear constrained elastostatic system. Asghari [32] developed a modified couple stress theory in a geometrical nonlinear micro-plate formulation to capture size effects. Belytschko et al. $[33,34]$ provided a complete descriptions for different stress formulations. 
In the literature, most of the works make use of the nonlinear von Kármán approximation. Furthermore, an Updated Lagrangian (UL) approach is often used with the assessment of corotational Cauchy stresses [35]. Stresses referred to the deformed configuration have a simpler physical interpretation because equilibrium equations express the balancing of internal stresses of the deformed state. However, as reported by Pai [1], the Lagrangian formulation is typically adopted in geometrical nonlinear problems because the structure, once unloaded, returns to its natural undeformed configuration. For nonlinear problems, strain and stress formulations need to be work-conjugate to employ in constitutive equations. Thus, a TL approach is more used that a UL formulation, since the accuracy of the current equilibrium condition does not depend on the previous solution, according to the iterative solution usually adopted for nonlinear problems. Moreover, the calculated displacements, stresses, and strains do not need any coordinate transformation at each iteration. Green-Lagrange strains and Second Piola-Kirchhoff (PK2) stresses are perfectly formulated in the TL approach. In addition, in order to perform stress analyses of geometrical nonlinear structures, PK2 stresses are sometimes transformed into true Cauchy's stresses, which are physically meaningful. Since the deformed configuration of many problems, especially those involving solids, is not known, it is not convenient to work with stress tensor, which is expressed in terms of spatial coordinates. In some cases, it is more convenient to deal with stress tensor that is referred to as the undeformed or an intermediate configuration. Even though the PK2 does not admit a physical interpretation there are three good reasons for using it as a measure of the forces acting on a material: (1) it is symmetric; (2) it is energy conjugate to the Green-Lagrange strain; (3) it is parametrized only by material coordinates.

The goal of this work is to show a new approach that, considering geometrical nonlinearities, can accurately calculate the 3D stress field. To perform these analyses, investigations are conducted by using CUF $[36,37]$. CUF is a hierarchical formulation in which the order of the structural model is considered as an input of the analysis so that no ad-hoc formulations are needed to achieve any refined generic models. As reported by Carrera et al. [2], any theory is degenerated into generalized kinematics using an arbitrary expansion of the generalized variables. With this procedure, the nonlinear governing equations and the relative FE arrays of the shell theories are formulated in terms of Fundamental Nuclei (FNs). FNs are the basic building blocks of the proposed formulation. CUF has been adopted for different engineering problems [38-41], and it has been extended to nonlinear geometric analyses, for the resolution of the static behaviour of metallic and composite structures [42-44] and in dynamics [45,46]. In a unified formulation, different expansion functions are considered to approximate the throughthe-thickness kinematics. The expansion functions and their order can be arbitrary (e.g., polynomials, exponentials, harmonic functions). Taylor (TE) and Lagrange (LE) polynomials are widely adopted as typical expansion functions. The unknowns of a TE model are displacements and $N$-order derivatives of the displacement field. On the other hand, the unknown variables become pure displacements if Lagrange polynomials are adopted as expansion functions.

This article is structured as follows: (i) firstly, preliminary information about the 2D CUF model are illustrated in Section 2, including the Green-Lagrange nonlinear geometrical relations and the FE approximation; (ii) then, Section 3 presents the method used to perform geometrical nonlinear analyses; (iii) numerical results are presented in Section 4, and they involve shell structures with various boundary and loading conditions; (iv) finally, conclusions are given in Section 5. 


\section{UNIFIED FINITE SHELL ELEMENT}

\subsection{Preliminaries}

A shell is a structural element where the thickness is smaller than the other dimensions. Typically, this geometry is represented by means of an orthogonal curvilinear reference system $(\alpha, \beta, z)$, where $\alpha$ and $\beta$ indicate the two in-plane directions and $z$ the through-the-thickness direction. A typical shell structure with curvatures and the reference system is depicted in Fig. 1.

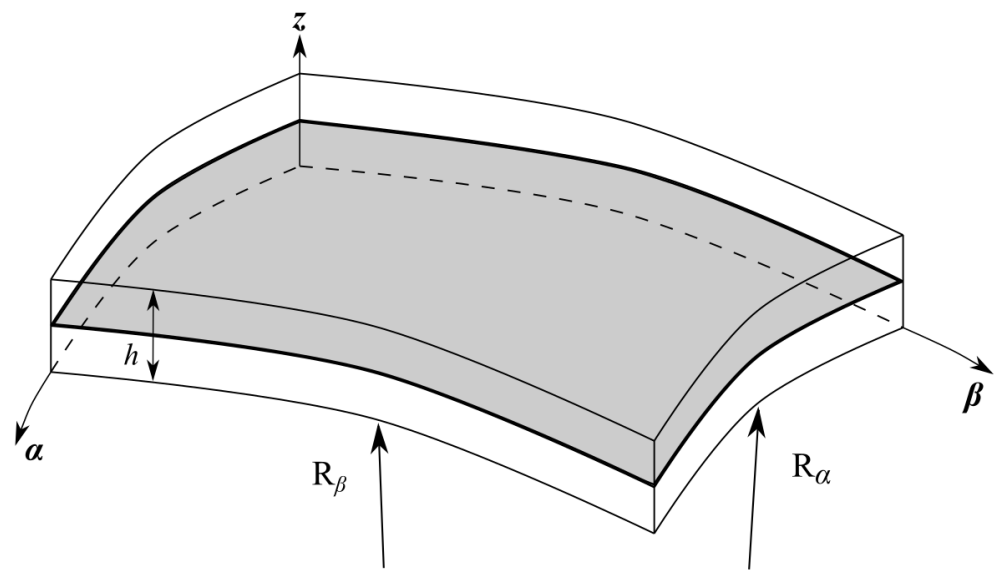

Fig. 1. Reference system of a generic shell

For the sake of brevity, the complete description of the shell formulation is not the purpose of this work, and interested readers are referred to [10,21,47].

For highly flexible shell structures, it is particularly important to consider high-order terms for an accurate description of their static behaviour. Considering only geometrical nonlinear problems, Lagrangian formulations are typically adopted. The advantage of using this formulation is that strains are written in terms of the undeformed configuration. For this reason, the TL formulation [35] is used in this work. In this domain, the Green-Lagrange strains $\boldsymbol{\epsilon}$, that is work-conjugate to the PK2 stress vector $S$ in the curvilinear reference system, is considered.

$$
\begin{aligned}
& \boldsymbol{\epsilon}=\left\{\epsilon_{\alpha \alpha}, \epsilon_{\beta \beta}, \epsilon_{z z}, \epsilon_{\alpha z}, \epsilon_{\beta z}, \epsilon_{\alpha \beta}\right\}^{T}, \\
& S=\left\{S_{\alpha \alpha}, S_{\beta \beta}, S_{z z}, S_{\alpha z}, S_{\beta z}, S_{\alpha \beta}\right\}^{T} .
\end{aligned}
$$

The displacement-strain relations are written as

$$
\boldsymbol{\epsilon}=\boldsymbol{\epsilon}_{l}+\boldsymbol{\epsilon}_{n l}=\left(\boldsymbol{b}_{l}+\boldsymbol{b}_{n l}\right) \boldsymbol{u},
$$

where $\boldsymbol{b}_{l}$ and $\boldsymbol{b}_{n l}$ are the linear and nonlinear differential operators, and $\boldsymbol{u}$ is the 3D displacement vector. These differential operators in the case of $2 \mathrm{D}$ model are reported in $[2,21]$. The displacement vector is defined as

$$
\boldsymbol{u}(\alpha, \beta, z)=\left\{u_{\alpha}, u_{\beta}, u_{z}\right\}^{T} .
$$

Using the constitutive equations, stresses are computed as

$$
S=C \epsilon,
$$

where the material elastic matrix $C$ is reported in Ref. [48, 49].

Therefore, in order to perform accurate nonlinear analyses and to evaluate reliable strains and stresses, a Lagrangian formulation is adopted and, in particular, the PK2 stress tensor is used. 


\subsection{CUF and FE approximations}

The 3D displacement field $\boldsymbol{u}(\alpha, \beta, z)$ of the proposed shell model is written, within the framework of the CUF, as a set of thickness functions depending only on the thickness coordinate $z$ and the corresponding variables depending on the in-plane coordinates $\alpha$ and $\beta$. Thus, we write

$$
\boldsymbol{u}(\alpha, \beta, z)=F_{\tau}(z) \boldsymbol{u}_{\tau}(\alpha, \beta), \quad \tau=0,1, \ldots, N
$$

where $F_{\tau}$ represents the expansion function of the thickness coordinate $z, \boldsymbol{u}_{\tau}(\alpha, \beta)$ denotes the generalized in-plane displacement vector and $N$ is the order of expansion in the thickness direction. The class of 2D CUF shell model adopted is determined by the arbitrary choice of $F_{\tau}$ and N; e.g., Taylor Expansions [50], Lagrange Expansions [51], Hierarchical Legendre Expansions [52].

In this article, LE and TE are considered as $F_{\tau}$ functions over the thickness direction. Carrera et al. [53] illustrated how the interfacial continuity of shear stresses using LE is opportunely obtained when the thickness functions are adequately refined. Furthermore, the proposed shell formulation adopts only displacement variables. For the sake of brevity, the adopted shell theories are denoted by the acronym LDN, which represent the Lagrange expansion, Displacementbased theory with the order N. In particular, the two-node linear (LD1), three-node quadratic (LD2), and the four-node cubic (LD3) LE functions are assumed in the z-direction in order to generate linear to higher-order kinematics CUF shell models with geometrical nonlinearities. On the other hand, the two-dimensional models based on the TE consider different base functions to model the displacement field of a structure along the thickness using polynomials of any order. The reader is referred to [37] for the complete expressions for both formulations.

For the sake of generality, when the FE discretization is introduced, the in-plane generalized displacement vector is approximated using the shape functions $N_{i}(\alpha, \beta)$ as

$$
\boldsymbol{u}_{\tau}(\alpha, \beta)=N_{i}(\alpha, \beta) \boldsymbol{q}_{\tau i}, \quad i=1,2, \ldots, n_{e l}
$$

where $\boldsymbol{q}_{\tau i}$ are the unknown nodal variables, $n_{e l}$ indicates the number of nodes per element and the index $i$ denotes summation.

For the sake of completeness, Fig. 2 illustrates the approximations of a typical shell structure adopting a 2D model. In this figure, the classical 2D four-node quadratic FE (Q4) and the 2D nine-node quadratic FE (Q9) are illustrated.

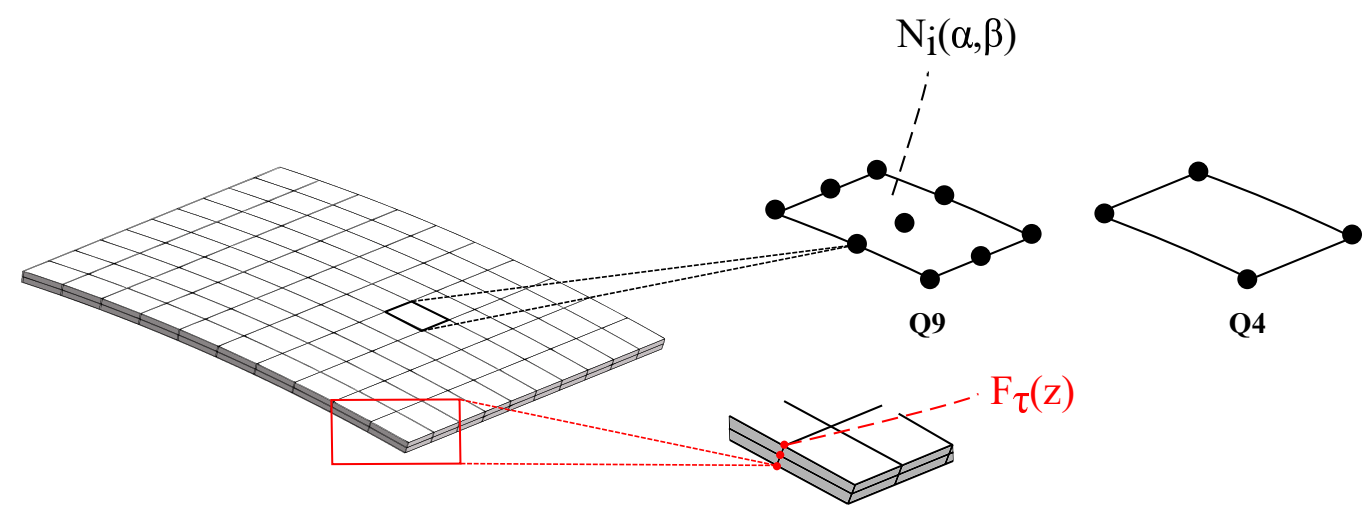

Fig. 2. The 2D model approximations of a typical shell structure

Q9 FE is the most widely used element for 2D model due to its high accuracy in analysis and flexibility in modelling. Since the goal of this work is to perform accurate stress analyses, the Q9 FE was used for the shape function in the $\alpha-\beta$ plane in the following evaluations. Readers are referred to [37] for a complete derivation of the shell FE formulations. 


\section{NONLINEAR GOVERNING EQUATIONS}

When large displacements and rotations occur, nonlinear analyses are necessary. The principle of virtual work is introduced to derive the nonlinear FE governing equations. Hence:

$$
\delta L_{\text {int }}=\delta L_{\text {ext }},
$$

where $\delta L_{\text {int }}$ and $\delta L_{\text {ext }}$ represent the virtual variation of the strain energy and the virtual variation of the work of external loads, respectively. To perform large deflection analyses of flexible structures, complex nonlinear differential problems should be solved. Since it is difficult to compute these problems, the nonlinear equilibrium equations of the shell structure based on the FEM and CUF can be written and solved as a system of nonlinear algebraic equations.

Considering the TL formulation and introducing the geometrical and constitutive relations, the virtual variation of the internal strain energy is formulated as (omitting some mathematical steps, which can be found in [21])

$$
\delta L_{\text {int }}=\int_{V} \delta \boldsymbol{\epsilon}^{T} \boldsymbol{\sigma} d V=\delta \boldsymbol{q}_{s j}^{T} \boldsymbol{K}_{S}^{i j \tau s} \boldsymbol{q}_{\tau i}
$$

where $\boldsymbol{K}_{S}^{i j \tau s}$ is the secant stiffness matrix. For the sake of brevity, the complete form of this matrix is omitted, see [21]. The virtual variation of the external work can be written as

$$
\delta L_{e x t}=\delta \boldsymbol{q}_{s j}^{T} \boldsymbol{p}_{s j},
$$

where $\boldsymbol{p}_{s j}$ represents the nodal loading vector, see [36] for its derivation. The nonlinear equilibrium conditions and the related finite element arrays of the generic shell theory is formulated as follows

$$
\boldsymbol{K}_{S}^{i j \tau s} \boldsymbol{q}_{i j}-\boldsymbol{p}_{s j}=0,
$$

This system constitutes a set of three nonlinear algebraic equations. Eq. (10) is arbitrarily expanded to obtain any desired theory by choosing the value for $\tau, s=1,2, \ldots, M$ and $i, j=$ $1,2, \ldots, p+1$, to give

$$
\boldsymbol{K}_{S} \boldsymbol{q}-\boldsymbol{p}=0,
$$

where $K_{S}, q$ and $p$ represent the global, assembled FE arrays of the final structure.

For the FE calculation of the nonlinear algebraic governing equations, an iterative method is needed. Usually, an incremental linearized scheme, typically the Newton-Raphson method is adopted to solve the geometrical nonlinear systems. According to the Newton-Raphson method, Eq. (11) is formulated as follows

$$
\boldsymbol{\varphi}_{\text {res }}=\boldsymbol{K}_{\mathrm{S}} \boldsymbol{q}-\boldsymbol{p}=0,
$$

where $\boldsymbol{\varphi}_{\text {res }}$ denotes the vector of the residual nodal forces (unbalanced nodal force vector). For the sake of brevity, the reader is referred to [54-56] for a complete description of this method.

\section{NUMERICAL RESULTS}

This section discusses representative benchmark problems, and particular emphasis is given to the potentiality of the proposed full geometrical nonlinear shell model to evaluate the 3D stress fields. Isotropic metallic shell structures are considered. At first, convergence analyses are shown, comparing the results with those provided from the literature. 1LD2 is chosen for the thickness expansion for the convergence analysis on the in-plane mesh approximations. Then, on the converged model, PK2 stress distributions are evaluated, and different expansions are adopted, from lower-order to higher-order. The considered cases are suggested by Sze's well-known paper [22]. The reference solutions reported in the following analyses are obtained using Abaqus. 


\subsection{Pinched cylindrical shell}

The first analysis deals with a clamped cylindrical shell under a pinching force at the free end. The vertical deflection and the rotation about the $\beta$-axis are restrained along its longitudinal edges. The model is reported in Fig. 3, along with its geometrical characteristics, $L=3.048 \mathrm{~m}, R_{\alpha}=$ $1.016 \mathrm{~m}$, and thickness equal to $0.03 \mathrm{~m}$. The material has an elastic modulus $E=2.0685 \times 10^{7} \mathrm{~N} / \mathrm{m}^{2}$ and a Poisson's ratio $v=0.3$.

This analysis case was presented by $\mathrm{Wu}$ et al. [21] and by Sze et al. [22]. Nevertheless, no through-the-thickness stress benchmarks were yet reported. The undeformed and deformed config-

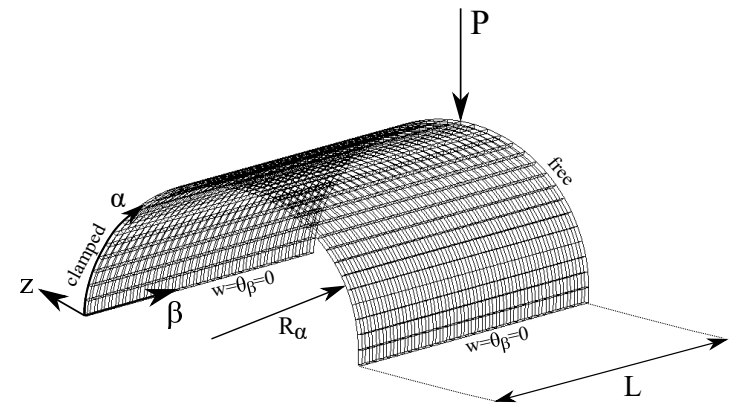

Fig. 3. Geometric properties, boundary and loading conditions of the pinched cylindrical shell urations of the pinched cylindrical considering a force of $P=2000 \mathrm{~N}$ in the linear analysis are shown in Fig. 4 .

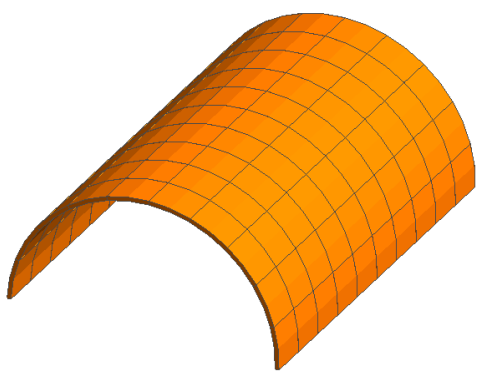

(a) Undeformed

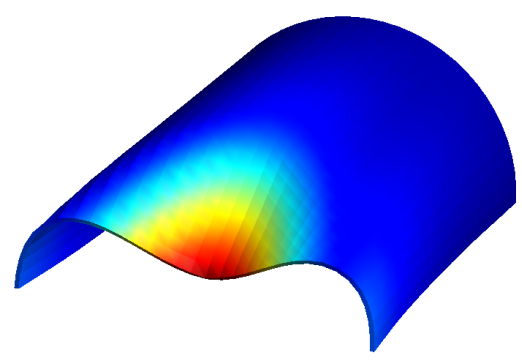

(b) Deformed

Fig. 4. Configurations of the pinched cylindrical shell under the point load $P=2000 \mathrm{~N}$ in the linear analysis

First, in order to perform an accurate static analysis, a convergence study on the in-plane finite element mesh is carried out. Fig. 5 shows the transverse deflection for different twodimensional shell models, and from 100Q9 to 1600Q9 elements are considered for the surface approximation, whereas one LD2 is used in the $z$-direction.

Moreover, Tab. 1 shows the transverse displacement values for different models and loads, along with the total degrees of freedom (DOFs). As evident from Fig. 5 and Tab. 1, the kinemat-

Table 1. Equilibrium points of nonlinear response curves at the load point of the pinched cylindrical shell for different geometrical nonlinear shell models

\begin{tabular}{crccc}
\hline \multirow{2}{*}{ Model } & \multirow{2}{*}{ DOFs } & \multicolumn{3}{c}{$-u_{z},[\mathrm{~m}]$} \\
\cline { 3 - 5 } & & $500 \mathrm{~N}$ & $1500 \mathrm{~N}$ & $2000 \mathrm{~N}$ \\
\hline $10 \times 10 \mathrm{Q} 9+1 \mathrm{LD} 2$ & 3969 & 0.34 & 0.73 & 1.24 \\
$16 \times 16 \mathrm{Q} 9+1 \mathrm{LD} 2$ & 9801 & 0.44 & 1.40 & 1.53 \\
$32 \times 16$ Q9 +1 LD2 & 19305 & 0.49 & 1.55 & 1.70 \\
$32 \times 32$ Q9 +1 LD2 & 38025 & 0.50 & 1.57 & 1.72 \\
$40 \times 40$ Q9 +1 LD2 & 59049 & 0.50 & 1.57 & 1.72 \\
\hline
\end{tabular}




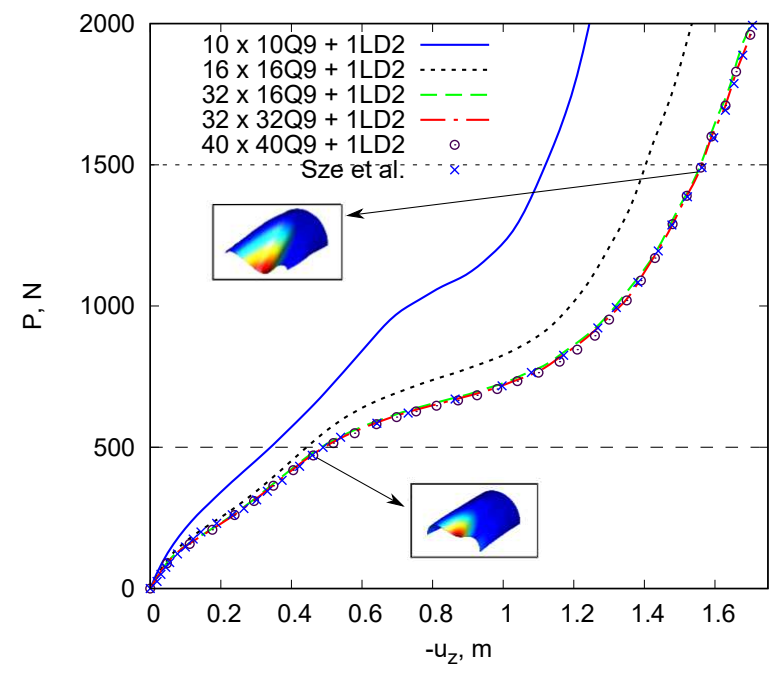

Fig. 5. Convergence study of nonlinear response curves for the isotropic pinched cylindrical shell.

Comparison of different in-plane mesh approximations

ics $32 \times 32$ Q9 is a reliable approximation for the in-plane mesh. In addition, for the evaluation of the 3D stress fields, including the circumferential normal stress $S_{\alpha \alpha}$ and the transverse shear stress $S_{\beta z}$, another convergence analysis on the expansion functions in the thickness direction is necessary. Both LE and TE functions are considered and compared in this analysis. Fig. 6 depicts the stress distributions for different expansion orders.
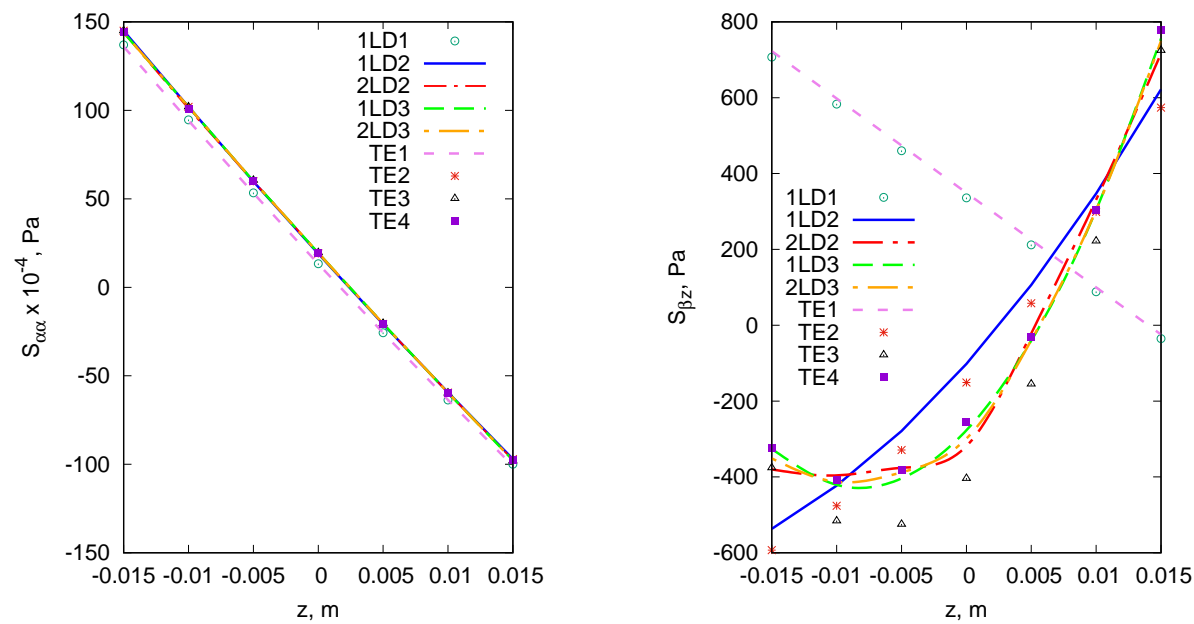

Fig. 6. Through-the-thickness PK2 stress distributions for different TE and LE functions of nonlinear response for the pinched cylindrical shell under the load $P=1500 \mathrm{~N}$. Values are evaluated at the middle point. $32 \times 32 \mathrm{Q} 9$ in-plane mesh model

The corresponding stress values are tabulated in Tab. 2, comparing the circumferential normal and the transverse shear PK2 stress values for different shell theories and loads.

Clearly, at least 1LD3 kinematics should be used to accurately predict the stress values. Results suggest that a low-order model is sufficient to evaluate the circumferential normal stress, whereas a high-order model is needed to accurately predict the transverse shear stress component. Furthermore, Fig. 7 shows circumferential normal and transverse shear stresses over the $z$-axis for two different loads. 
Table 2. Circumferential normal stress and transverse shear stress values of nonlinear response curves of the pinched cylindrical shell (with $32 \times 32 \mathrm{Q} 9$ in-plane mesh) for different expansion theories and loads at $\alpha=1.595 \mathrm{~m}, \beta=1.524 \mathrm{~m}$ and $z=0.015 \mathrm{~m}$

\begin{tabular}{ccrrrrr}
\hline \multirow{2}{*}{ Theory } & \multirow{2}{*}{ DOFs } & \multicolumn{2}{c}{$S_{\alpha \alpha} \times 10^{-4},[\mathrm{~Pa}]$} & & \multicolumn{2}{c}{$S_{\beta z},[\mathrm{~Pa}]$} \\
\cline { 3 - 4 } \cline { 6 - 6 } & & $500 \mathrm{~N}$ & $1500 \mathrm{~N}$ & & $500 \mathrm{~N}$ & $1500 \mathrm{~N}$ \\
\hline TE1 & 25350 & -10.72 & -100.75 & & -704.74 & -24.28 \\
TE2 & 38025 & -9.80 & -97.12 & & -748.48 & 573.55 \\
TE3 & 50700 & -9.85 & -97.88 & & 34.82 & 724.84 \\
TE4 & 63375 & -9.85 & -97.87 & & 29.49 & 777.75 \\
1LD1 & 25350 & -10.72 & -100.74 & & -908.82 & -35.38 \\
1LD2 & 38025 & -9.80 & -97.11 & & -760.31 & 622.33 \\
2LD2 & 63375 & -9.84 & -97.62 & & -173.83 & 717.30 \\
1LD3 & 50700 & -9.85 & -97.87 & & 29.53 & 756.33 \\
2LD3 & 88725 & -9.85 & -97.87 & & 29.67 & 755.49 \\
\hline
\end{tabular}

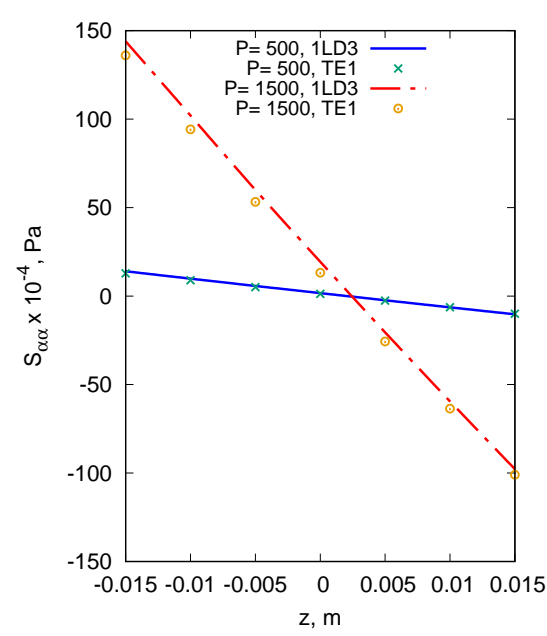

(a)

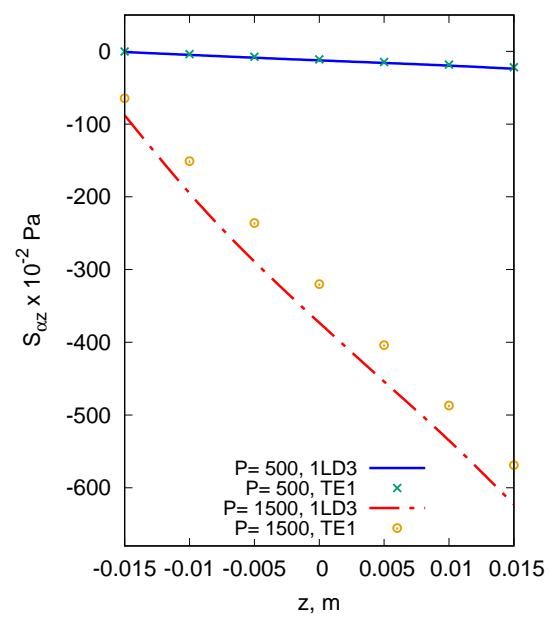

(c)

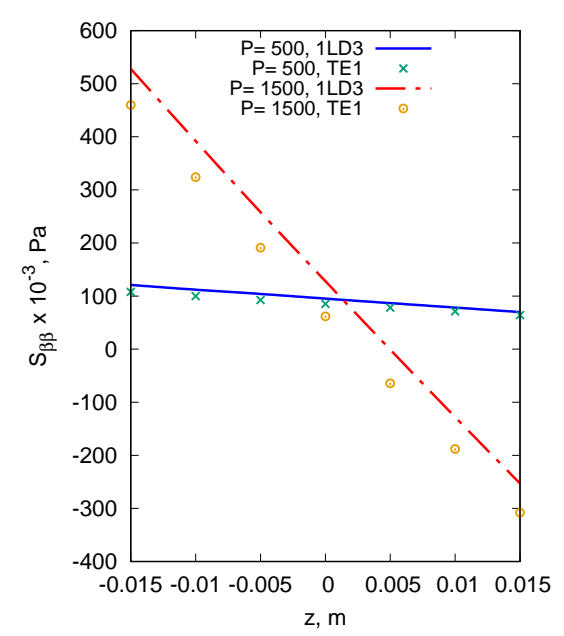

(b)

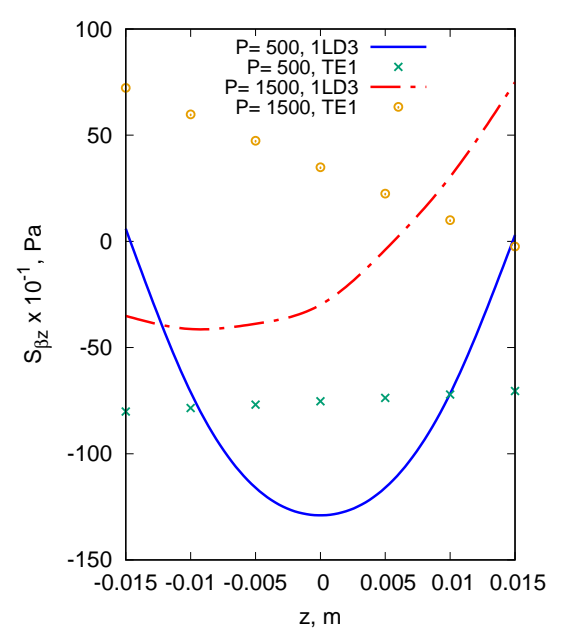

(d)

Fig. 7. Through-the-thickness distributions of normal $(a, b)$ and transverse shear $(c, d)$ PK2 stresses for two different loads. Values are evaluated at the middle point. Pinched cylindrical shell 
The stress distributions of both TE of order one (TE1) and one LD3 shell models are reported to highlight the different capabilities of the two CUF shell theories in catching accurate stress distributions. The linear interpolation provided by the TE1 approximation is not enough to catch the parabolic distribution of the transverse shear stress, as underlined in Fig. 7(d). Indeed, it needs to consider at least 1LD3 for a proper description of the given problem.

\subsection{Hinged cylindrical shell}

The following case deals with a hinged cylindrical shell under a central transverse force $P$, as illustrated in Fig. 8. This nonlinear benchmark problem is very popular due to the snapping behaviour, which means that the tangent global stiffness matrices become singular at some intermediate cases $[57,58]$. The material properties of the isotropic cylindrical shell are $E=$ $3102.75 \mathrm{MPa}$ and $v=0.3$, with $L=508 \mathrm{~mm}, R_{\alpha}=2540 \mathrm{~mm}, \theta=0.1 \mathrm{rad}$ and thickness equal to $12.7 \mathrm{~mm}$. All nodal displacements are restrained along the hinged edges.

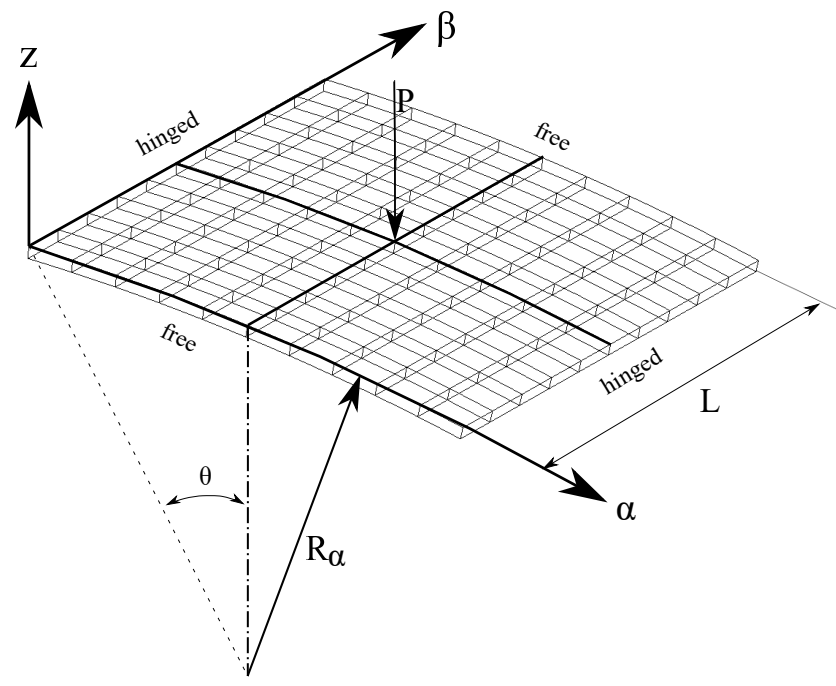

Fig. 8. Hinged cylindrical shell under a central transverse force

The undeformed and deformed configurations in the linear analysis are shown in Fig. 9.

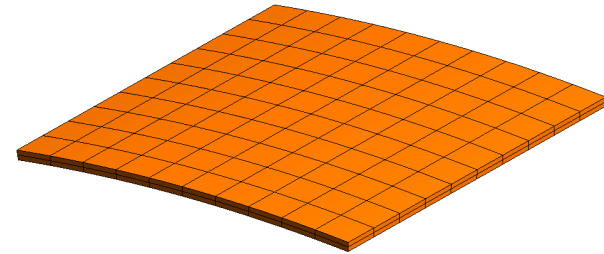

(a) Undeformed

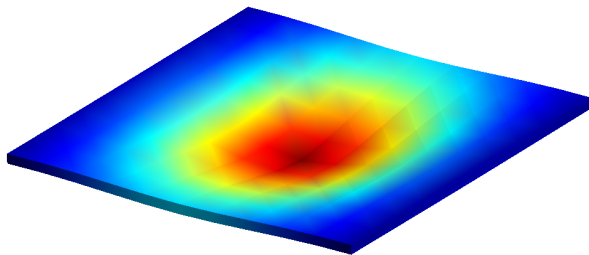

(b) Deformed

Fig. 9. Configurations of the hinged isotropic cylindrical shell under the point load $P=3000 \mathrm{~N}$ in the linear analysis

First, in order to perform an accurate static evaluation, convergence analysis of the in-plane 2D shell model is carried out. Then, a stress evaluation is performed for different expansion orders. Fig. 10 plots the transverse deflection for different in-plane finite element mesh. From 25Q9 to 225Q9 elements are used for the surface approximation, whereas one LD2 is adopted over the $z$-direction. The nonlinear response curves are divided into three regions $\mathrm{A}, \mathrm{B}$ and $\mathrm{C}$.

In addition, transverse displacements for different in-plane meshes and loads are reported in Tab. 3, along with the number of DOFs. 


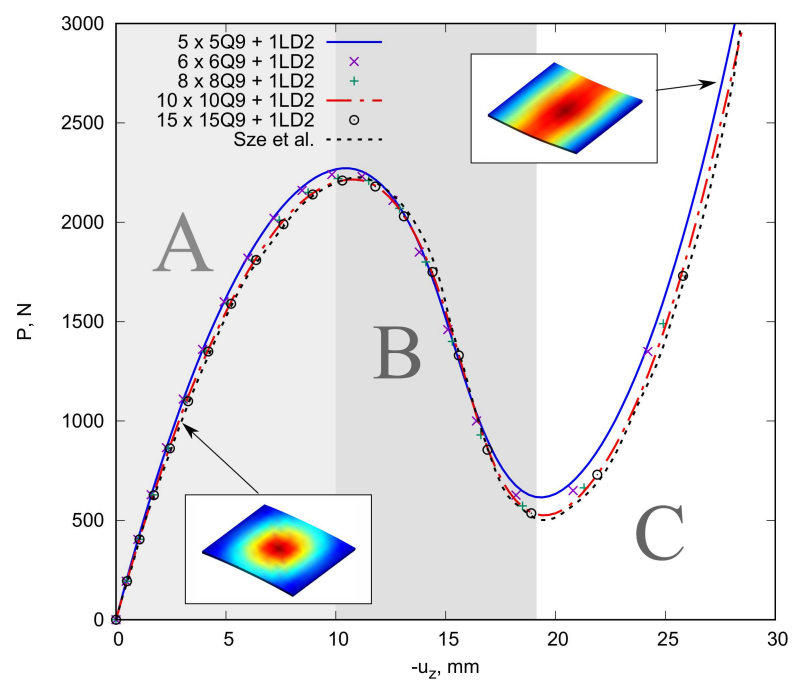

Fig. 10. Convergence study of nonlinear response curves for the hinged cylindrical shell under the point load. Comparison of different in-plane mesh approximations

Table 3. Equilibrium points of nonlinear response curves of the hinged cylindrical shell for different surface mesh approximations and loads at $\alpha=254.0 \mathrm{~mm}, \beta=254.0 \mathrm{~mm}$ and $z=0 \mathrm{~mm}$

\begin{tabular}{|c|c|c|c|c|c|c|c|c|c|c|}
\hline \multirow{2}{*}{ Model } & \multirow{2}{*}{ DOFs } & \multicolumn{3}{|c|}{$-u_{z}[\mathrm{~mm}]$ in $\mathrm{A}$} & \multicolumn{3}{|c|}{$-u_{z}[\mathrm{~mm}]$ in B } & \multicolumn{3}{|c|}{$-u_{z}[\mathrm{~mm}]$ in $C$} \\
\hline & & $1000 \mathrm{~N}$ & $1500 \mathrm{~N}$ & $2000 \mathrm{~N}$ & $1000 \mathrm{~N}$ & $1500 \mathrm{~N}$ & $2000 \mathrm{~N}$ & $1000 \mathrm{~N}$ & $1500 \mathrm{~N}$ & $2000 \mathrm{~N}$ \\
\hline $5 \times 5 \mathrm{Q} 9+1 \mathrm{LD} 2$ & 726 & 2.71 & 4.51 & 7.03 & 17.76 & 16.25 & 13.10 & 22.23 & 24.35 & 25.72 \\
\hline $6 \times 6 \mathrm{Q} 9+1 \mathrm{LD} 2$ & 1521 & 2.72 & 4.52 & 7.08 & 18.18 & 13.96 & 13.31 & 22.51 & 24.56 & 25.71 \\
\hline $8 \times 8 Q 9+1 \mathrm{LD} 2$ & 2601 & 2.82 & 4.79 & 7.39 & 15.51 & 14.43 & 13.80 & 22.75 & 24.92 & 26.02 \\
\hline $10 \times 10 \mathrm{Q} 9+1 \mathrm{LD} 2$ & 3969 & 2.89 & 4.81 & 7.65 & 15.70 & 14.79 & 14.10 & 22.91 & 24.89 & 26.29 \\
\hline $15 \times 15 \mathrm{Q} 9+1 \mathrm{LD} 2$ & 8649 & 2.90 & 4.80 & 7.66 & 15.71 & 14.80 & 14.11 & 22.92 & 24.88 & 26.30 \\
\hline
\end{tabular}
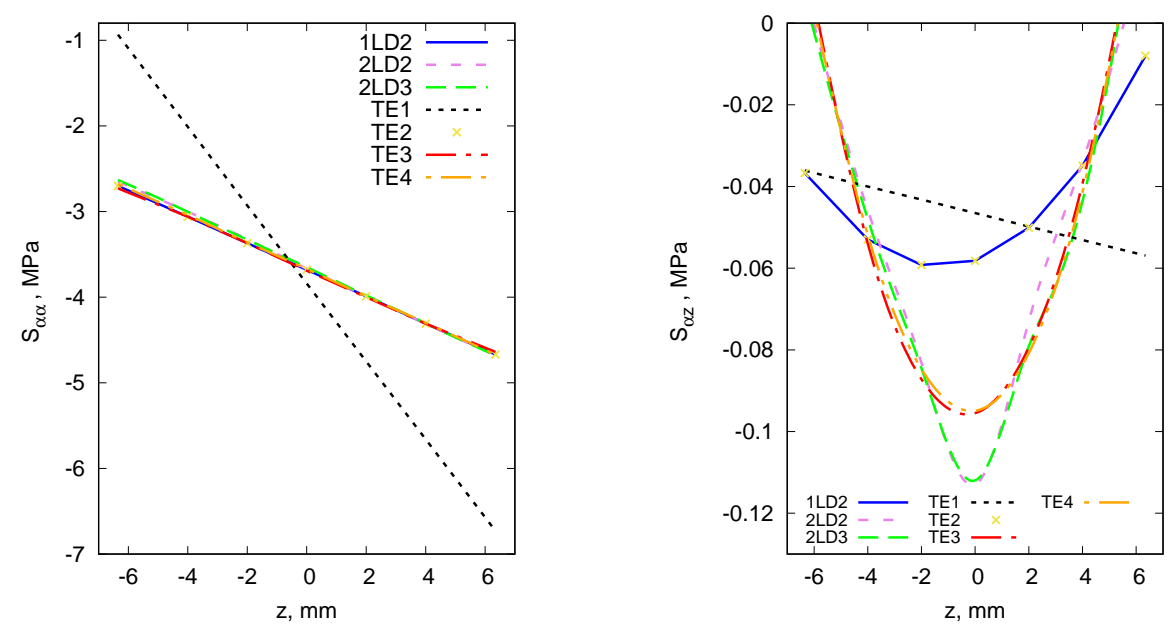

Fig. 11. Through-the-thickness PK2 stress distributions for different LE and TE functions of the hinged cylindrical shell under a point load, $P=2000 \mathrm{~N}$ in region $\mathrm{A}$, at $\alpha=127.0 \mathrm{~mm}$ and $\beta=254.0 \mathrm{~mm}$.

$10 \times 10$ Q9 in-plane mesh model

Consequently, the kinematics $10 \times 10 \mathrm{Q} 9$ will be taken as converged discretization and, therefore, it is used for the stress evaluation. To perform an accurate stress prediction, different 
expansion functions in the z-direction are compared. Both LE and TE functions are considered in this analysis. Fig. 11 shows the comparison for different through-the-thickness kinematic theories for the stress assessment.

The corresponding stress values at $\alpha=127.0 \mathrm{~mm}, \beta=254.0 \mathrm{~mm}$ and $z=6.35 \mathrm{~mm}$ for circumferential normal stress and $z=0 \mathrm{~mm}$ for transverse shear stress are tabulated in Tab. 4 for different shell theories.

Table 4. Circumferential normal PK2 stress and transverse shear PK2 stress values of nonlinear analyses of the hinged cylindrical shell under a $P=2000 \mathrm{~N}$ (with $10 \times 10$ Q9 in-plane mesh) for different CUF shell theories at $\alpha=127.0 \mathrm{~mm}$ and $\beta=254.0 \mathrm{~m}$

\begin{tabular}{cccc}
\hline Theory & DOFs & $S_{\alpha \alpha}\left[\mathrm{N} / \mathrm{mm}^{2}\right]$ in A at $z=6.35 \mathrm{~mm}$ & $S_{\alpha z}\left[\mathrm{~N} / \mathrm{mm}^{2}\right]$ in A at $z=0 \mathrm{~mm}$ \\
\hline TE1 & 2646 & -6.73 & -0.046 \\
TE2 & 3969 & -4.67 & -0.058 \\
TE3 & 5292 & -4.67 & -0.095 \\
TE4 & 6615 & -4.67 & -0.095 \\
TE8 & 11907 & -4.67 & -0.096 \\
1LD2 & 3969 & -4.67 & -0.058 \\
2LD2 & 6615 & -4.67 & -0.112 \\
2LD3 & 9261 & -4.67 & -0.112 \\
\hline
\end{tabular}

In addition, results at $\alpha=254.0 \mathrm{~mm}, \beta=254.0 \mathrm{~mm}$ and $z=6.35 \mathrm{~mm}$ are reported in Tab. 5, showing the circumferential normal PK2 stress values for different CUF shell theories and loads.

Table 5. Circumferential normal PK2 stress values of nonlinear response curves of the hinged cylindrical shell (with 10×10 Q9 in-plane mesh) for different CUF shell theories and loads at $\alpha=254.0 \mathrm{~mm}, \beta=254.0 \mathrm{~mm}$ and $z=6.35 \mathrm{~mm}$

\begin{tabular}{|c|c|c|c|c|c|c|c|}
\hline \multirow{2}{*}{ Theory } & \multirow{2}{*}{ DOFs } & \multicolumn{2}{|c|}{$S_{\alpha \alpha}\left[\mathrm{N} / \mathrm{mm}^{2}\right]$ in $\mathrm{A}$} & \multicolumn{2}{|c|}{$S_{\alpha \alpha}\left[\mathrm{N} / \mathrm{mm}^{2}\right]$ in B } & \multicolumn{2}{|c|}{$S_{\alpha \alpha}\left[\mathrm{N} / \mathrm{mm}^{2}\right]$ in C } \\
\hline & & $1000 \mathrm{~N}$ & $2000 \mathrm{~N}$ & $1000 \mathrm{~N}$ & $2000 \mathrm{~N}$ & $1000 \mathrm{~N}$ & $2000 \mathrm{~N}$ \\
\hline TE1 & 2646 & -12.82 & -29.81 & -26.69 & -31.07 & -28.24 & -36.20 \\
\hline TE2 & 3969 & -14.08 & -30.24 & -28.98 & -36.46 & -30.13 & -38.56 \\
\hline TE3 & 5292 & -14.33 & -30.07 & -29.29 & -36.89 & -30.34 & -38.97 \\
\hline TE4 & 6615 & -14.25 & -30.59 & -29.09 & -36.65 & -30.27 & -38.85 \\
\hline TE8 & 11907 & -14.25 & -30.60 & -29.07 & -36.63 & -30.26 & -38.86 \\
\hline 1LD2 & 3969 & -13.63 & -30.24 & -29.98 & -36.46 & -30.13 & -38.56 \\
\hline 2LD2 & 6615 & -14.28 & -30.70 & -29.06 & -36.67 & -30.27 & -38.90 \\
\hline 2LD3 & 9261 & -14.28 & -30.69 & -29.05 & -36.68 & -30.26 & -38.91 \\
\hline
\end{tabular}

Clearly, at least 2LD2 kinematics should be exploited to predict the stress values accurately. Fig. 12 depicts the circumferential normal and transverse shear PK2 stresses over the $z$-axis for different loads.

As previously reported, the PK2 stresses obtained using TE1 formulations are also reported in these graphs. According to Fig. 12, using a TE1 shell theory cannot be able to accurately predict the PK2 stresses, especially if the transverse shear stresses are evaluated. 


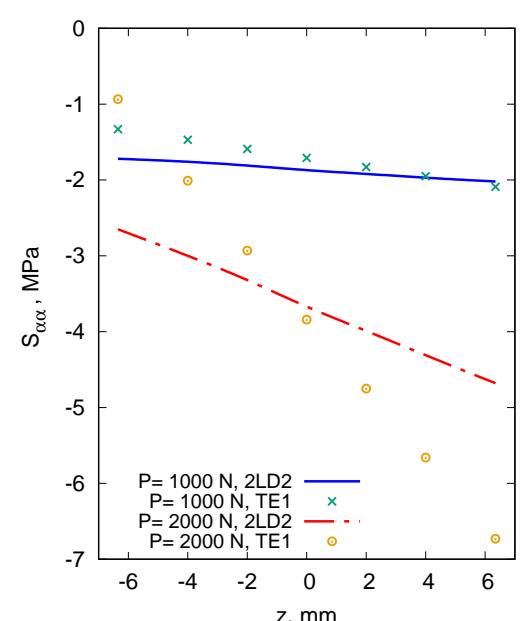

(a)

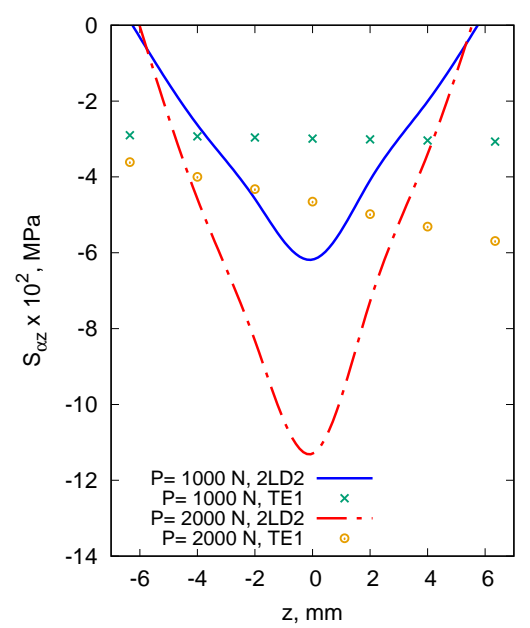

(c)

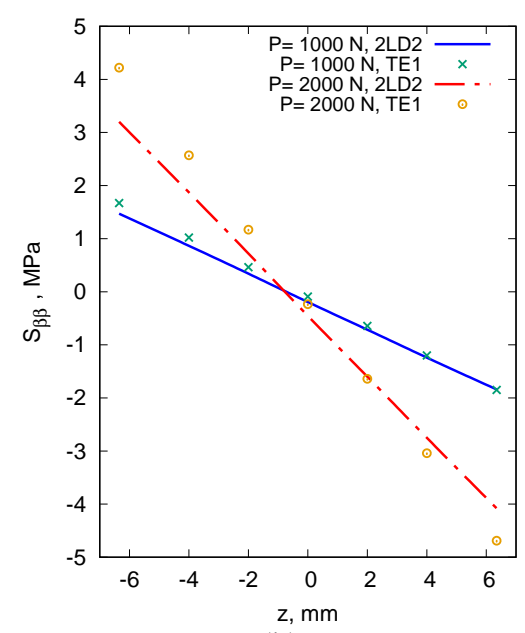

(b)

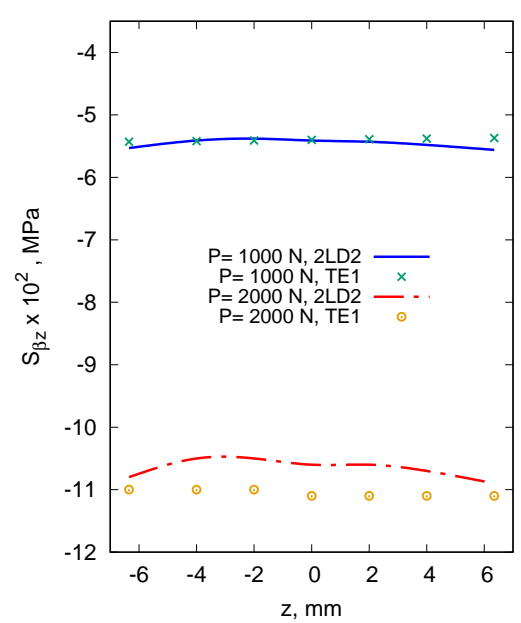

(d)

Fig. 12. Through-the-thickness distribution of normal $(a, b)$ and transverse shear $(c, d)$ PK2 stresses for two different loads in region $\mathrm{A}$ at $\alpha=127.0 \mathrm{~mm}$ and $\beta=254.0 \mathrm{~mm}$. Hinged cylindrical shell with $10 \times 10 \mathrm{Q} 9$ model.

\section{CONCLUSIONS}

The purpose of this paper was to provide accurate three-dimensional (3D) stress fields within cylindrical structures using two-dimensional (2D) shell models. A full geometrical nonlinear refined shell theory based on the Carrera Unified Formulation (CUF) and a Total Lagrangian (TL) approach has been adopted. The nonlinear governing equations and the related finite element arrays have been expressed by means of the principle of virtual work. The analyzed cases have been studied by employing a path-following Newton-Raphson linearized incremental scheme employing an arc-length constraint to compute the nonlinear algebraic system. Nonlinear static analyses of typical benchmark shell structures have been performed to obtain the load-deflection curves and, in particular, the stress distributions. Convergence analyses have been carried out for several in-plane mesh approximations and different expansion functions in the $z$-direction. Both Lagrange functions and Taylor polynomials have been considered for the implementation of low- to high-order structural models and compared to show the different stress results that can be obtained. The Second Piola-Kirchhoff (PK2) stresses 
have been reported to provide a stress benchmark for future comparisons. The results suggest that refined models are able to deal with the large deflection and to rigorously evaluate the stress distributions using various shell theories. Furthermore, it is shown that for the normal stress components, a lower-order CUF shell model is sufficient, whereas to accurately predict transverse shear stresses, a higher-order model is demanded.

\section{REFERENCES}

[1] P. F. Pai. Highly flexible structures: Modeling, computation, and experimentation. AIAA Education Series, Reston, VA, (2007).

[2] E. Carrera, A. Pagani, R. Augello, and B. Wu. Popular benchmarks of nonlinear shell analysis solved by 1D and 2D CUF-based finite elements. Mechanics of Advanced Materials and Structures, (2020), pp. 1-12. https://doi.org/10.1080/15376494.2020.1728450.

[3] S. D. Poisson. Mémoire sur l'équilibre et le mouvement des corps élastiques. Mm. Acad. Sci. Instr. Fr., 8, (1829), pp. 357-570.

[4] A. E. H. Love. Mathematical theory of elasticity. Cambridge University Press, (2013).

[5] R. D. Mindlin. Influence of rotatory inertia and shear flexural motions of isotropic elastic plates. Journal of Applied Mechanics, 18, (1951), pp. 1031-1036.

[6] G. Kirchhoff. Uber da gleichgewicht und die bewegung einer elastischen scheibe. Journal für die reine und angewandte Mathematik, 40, (1850), pp. 51-88.

[7] E. Reissner. The effect of transverse shear deformation on the bending of elastic plates. Journal of Applied Mechanics, 12, (1945), pp. 69-76.

[8] A. L. Cauchy. Sur l'equilibre et le mouvement d'une plaque solide. Exercises de Matematique, 3, (1828), pp. 328-355.

[9] W. T. Koiter. On foundations of linear theory of thin elastic shells. In Proceedings of the Koninklijke Nederlandse Akademie van Wetenschappen Series B-Physical Sciences, Vol. 73, (1970), pp. 169-195.

[10] P. M. Naghdi. Theory of shells and plates. Linear Theories of Elasticity and Thermoelasticity, (1973), pp. 425-640, https:/ / doi.org/10.1007/978-3-662-39776-3_5.

[11] M. Petrolo and E. Carrera. Methods and guidelines for the choice of shell theories. Acta Mechanica, 231, (2), (2020), pp. 395-434. https://doi.org/10.1007/s00707-019-02601-w.

[12] J. N. Reddy and C. F. Liu. A higher-order shear deformation theory of laminated elastic shells. International Journal of Engineering Science, 23, (3), (1985), pp. 319-330. https://doi.org/10.1016/00207225(85)90051-5.

[13] J. N. Reddy. A simple higher-order theory for laminated composite plates. Journal of Applied Mechanics, 51, (4), (1984), pp. 745-752. https://doi.org/10.1115/1.3167719.

[14] J. N. Reddy. Mechanics of laminated composite plates and shells: Theory and analysis. New York: CRC Press, (2004).

[15] E. Carrera. Theories and finite elements for multilayered, anisotropic, composite plates and shells. Archives of Computational Methods in Engineering, 9, (2), (2002), pp. 87-140. https://doi.org/10.1007/bf02736649.

[16] E. Carrera. Theories and finite elements for multilayered plates and shells: A unified compact formulation with numerical assessment and benchmarking. Archives of Computational Methods in Engineering, 10, (3), (2003), pp. 215-296. https://doi.org/10.1007/bf02736224.

[17] M. Cinefra and E. Carrera. Shell finite elements with different through-the-thickness kinematics for the linear analysis of cylindrical multilayered structures. International Journal for Numerical Methods in Engineering, 93, (2), (2013), pp. 160-182. https://doi.org/10.1002/nme.4377.

[18] M. Cinefra and S. Valvano. A variable kinematic doubly-curved MITC9 shell element for the analysis of laminated composites. Mechanics of Advanced Materials and Structures, 23, (11), (2016), pp. 1312-1325. https://doi.org/10.1080/15376494.2015.1070304.

[19] G. Li, E. Carrera, M. Cinefra, A. G. de Miguel, A. Pagani, and E. Zappino. An adaptable refinement approach for shell finite element models based on node-dependent kinematics. Composite Structures, 210, (2019), pp. 1-19. https://doi.org/10.1016/j.compstruct.2018.10.111.

[20] A. Pagani, E. Carrera, and R. Augello. Evaluation of various geometrical nonlinearities in the response of beams and shells. AIAA Journal, 57, (8), (2019), pp. 3524-3533. https://doi.org/10.2514/1.j057877. 
[21] B. Wu, A. Pagani, W. Q. Chen, and E. Carrera. Geometrically nonlinear refined shell theories by Carrera Unified Formulation. Mechanics of Advanced Materials and Structures, (2019), pp. 1-21. https://doi.org/10.1080/15376494.2019.1702237.

[22] K. Y. Sze, X. H. Liu, and S. H. Lo. Popular benchmark problems for geometric nonlinear analysis of shells. Finite Elements in Analysis and Design, 40, (2004), pp. 1551-1569. https://doi.org/10.1016/j.finel.2003.11.001.

[23] A. N. Palazotto. Nonlinear analysis of shell structures. AIAA Series, (1992).

[24] L. S. Ma and T. J. Wang. Nonlinear bending and post-buckling of a functionally graded circular plate under mechanical and thermal loadings. International Journal of Solids and Structures, 40, (1314), (2003), pp. 3311-3330. https://doi.org/10.1016/s0020-7683(03)00118-5.

[25] E. Carrera and H. Parisch. An evaluation of geometrical nonlinear effects of thin and moderately thick multilayered composite shells. Composite Structures, 40, (1), (1997), pp. 11-24. https://doi.org/10.1016/s0263-8223(97)00145-1.

[26] T. J. R. Hughes and W. K. Liu. Nonlinear finite element analysis of shells: Part I. Threedimensional shells. Computer Methods in Applied Mechanics and Engineering, 26, (3), (1981), pp. 331362. https://doi.org/10.1016/0045-7825(81)90121-3.

[27] K. S. Surana. Geometrically nonlinear formulation for the curved shell elements. International Journal for Numerical Methods in Engineering, 19, (4), (1983), pp. 581-615. https://doi.org/10.1002/nme.1620190409.

[28] S. J. Lee and W. Kanok-Nukulchai. A nine-node assumed strain finite element for large-deformation analysis of laminated shells. International Journal for Numerical Methods in Engineering, 42, (5), (1998), pp. 777-798.

[29] Y. Ko, P. S. Lee, and K. J. Bathe. The MITC4 shell element in geometric nonlinear analysis. Computers $\mathcal{E}$ Structures, 185, (2017), pp. 1-14. https://doi.org/10.1016/j.compstruc.2017.01.015.

[30] W. Flügge. Stresses in shells. Springer Science \& Business Media, (2013).

[31] D. A. Tortorelli. Sensitivity analysis for non-linear constrained elastostatic systems. International Journal for Numerical Methods in Engineering, 33, (8), (1992), pp. 1643-1660. https://doi.org/10.1002/nme.1620330807.

[32] M. Asghari. Geometrically nonlinear micro-plate formulation based on the modified couple stress theory. International Journal of Engineering Science, 51, (2012), pp. 292-309. https://doi.org/10.1016/j.ijengsci.2011.08.013.

[33] T. Belytschko, W. K. Liu, B. Moran, and K. Elkhodary. Nonlinear finite elements for continua and structures. John Wiley \& Sons, (2013).

[34] R. M. Hackett. Hyperelasticity primer. Springer, (2016).

[35] K. J. Bathe and S. Bolourchi. Large displacement analysis of three-dimensional beam structures. International Journal for Numerical Methods in Engineering, 14, (7), (1979), pp. 961-986. https://doi.org/10.1002/nme.1620140703.

[36] E. Carrera, G. Giunta, and M. Petrolo. Beam structures: Classical and advanced theories. John Wiley \& Sons, (2011).

[37] E. Carrera, M. Cinefra, M. Petrolo, and E. Zappino. Finite element analysis of structures through unified formulation. John Wiley \& Sons, (2014).

[38] E. Carrera, M. Filippi, P. K. R. Mahato, and A. Pagani. Accurate static response of single-and multi-cell laminated box beams. Composite Structures, 136, (2016), pp. 372-383. https://doi.org/10.1016/j.compstruct.2015.10.020.

[39] M. Filippi and E. Carrera. Capabilities of 1D CUF-based models to analyse metallic/composite rotors. Advances in Aircraft and Spacecraft Science, 3, (1), (2016), pp. 1-14. https://doi.org/10.12989/aas.2016.3.1.001.

[40] E. Carrera and A. Pagani. Free vibration analysis of civil engineering structures by component-wise models. Journal of Sound and Vibration, 333, (19), (2014), pp. 4597-4620. https://doi.org/10.1016/j.jsv.2014.04.063.

[41] A. Pagani, M. Petrolo, G. Colonna, and E. Carrera. Dynamic response of aerospace structures by means of refined beam theories. Aerospace Science and Technology, 46, (2015), pp. 360-373. https://doi.org/10.1016/j.ast.2015.08.005. 
[42] A. Pagani and E. Carrera. Unified formulation of geometrically nonlinear refined beam theories. Mechanics of Advanced Materials and Structures, 25, (1), (2018), pp. 15-31. https://doi.org/10.1080/15376494.2016.1232458.

[43] A. Pagani and E. Carrera. Large-deflection and post-buckling analyses of laminated composite beams by Carrera Unified Formulation. Composite Structures, 170, (2017), pp. 40-52. https://doi.org/10.1016/j.compstruct.2017.03.008.

[44] B. Wu, A. Pagani, M. Filippi, W. Q. Chen, and E. Carrera. Large-deflection and post-buckling analyses of isotropic rectangular plates by Carrera Unified Formulation. International Journal of NonLinear Mechanics, 116, (2019), pp. 18-31. https://doi.org/10.1016/j.ijnonlinmec.2019.05.004.

[45] A. Pagani, R. Augello, and E. Carrera. Frequency and mode change in the large deflection and postbuckling of compact and thin-walled beams. Journal of Sound and Vibration, 432, (2018), pp. 88-104. https://doi.org/10.1016/j.jsv.2018.06.024.

[46] E. Carrera, A. Pagani, and R. Augello. Effect of large displacements on the linearized vibration of composite beams. International Journal of Non-Linear Mechanics, 120, (2020). https://doi.org/10.1016/j.ijnonlinmec.2019.103390.

[47] A. E. Green and W. Zerna. Theoretical elasticity. Courier Corporation, (1992).

[48] K. J. Bathe. Finite element procedure. Prentice Hall, Upper Saddle River, New Jersey, USA, (1996).

[49] T. J. R. Hughes. The finite element method: Linear static and dynamic finite element analysis. Courier Corporation, (2012).

[50] E. Carrera and G. Giunta. Refined beam theories based on a unified formulation. International Journal of Applied Mechanics, 2, (1), (2010), pp. 117-143. https://doi.org/10.1142/S1758825110000500.

[51] E. Carrera and M. Petrolo. Refined beam elements with only displacement variables and plate/shell capabilities. Meccanica, 47, (3), (2012), pp. 537-556. https://doi.org/10.1007/s11012-011-9466-5.

[52] E. Carrera, A. G. de Miguel, and A. Pagani. Hierarchical theories of structures based on Legendre polynomial expansions with finite element applications. International Journal of Mechanical Sciences, 120, (2017), pp. 286-300. https://doi.org/10.1016/j.ijmecsci.2016.10.009.

[53] E. Carrera, M. Cinefra, G. Li, and G. M. Kulikov. MITC9 shell finite elements with miscellaneous through-the-thickness functions for the analysis of laminated structures. Composite Structures, 154, (2016), pp. 360-373. https://doi.org/10.1016/j.compstruct.2016.07.032.

[54] J. N. Reddy. An introduction to nonlinear finite element analysis: with applications to heat transfer, fluid mechanics, and solid mechanics. Oxford University Press, Oxford, (2014).

[55] E. Carrera. A study on arc-length-type methods and their operation failures illustrated by a simple model. Computers E Structures, 50, (2), (1994), pp. 217-229. https://doi.org/10.1016/00457949(94)90297-6.

[56] M. A. Crisfield. A fast incremental/iterative solution procedure that handles "snap-through". Computers $\mathcal{E}$ Structures, 13, (1), (1981), pp. 55-62. https://doi.org/10.1016/b978-0-08-027299-3.50009-1.

[57] J. A. T. Barbosa and A. J. M. Ferreira. Geometrically nonlinear analysis of functionally graded plates and shells. Mechanics of Advanced Materials and Structures, 17, (1), (2009), pp. 40-48. https://doi.org/10.1080/15376490903082870.

[58] D. S. Jagannathan, P. P. Christiano, and H. I. Epstein. Nonlinear analysis of reticulated space trusses. Journal of the Structural Division, 101, (12), (1975), pp. 2641-2658. 